\title{
ANALYSIS WITH WEAK TRACE IDEALS AND THE NUMBER OF BOUND STATES OF SCHRÖDINGER OPERATORS
}

\author{
BY \\ BARRY SIMON( $\left.{ }^{1}\right)$
}

\begin{abstract}
We discuss interpolation theory for the operator ideals $I_{p}^{w}$ defined on a separable Hilbert space as those operators $A$ whose singular values $\mu_{n}(A)$ obey $\mu_{n} \leqslant c n^{-1 / p}$ for some $c$. As an application we consider the functional $N(V)=\operatorname{dim}$ (spectral projection on $(-\infty, 0)$ for $-\Delta+V)$ on functions $V$ on $\mathrm{R}^{n}, n \geqslant 3$. We prove that for any $\epsilon>0: N(V) \leqslant C_{\epsilon}\left(\|V\|_{n / 2+\epsilon}+\|V\|_{n / 2-\epsilon}\right)^{n / 2}$ where $\|\cdot\|_{p}$ is an $L^{p}$ norm and that $\lim _{\lambda \rightarrow \infty} N(\lambda V) / \lambda^{n / 2}=(2 \pi)^{-n} \tau_{n} \int\left|V_{-}(x)\right|^{n / 2} d^{n} x$ for any $V \in L^{n / 2-\epsilon} \cap L^{n / 2+\epsilon}$. Here $V_{-}$is the negative part of $V$ and $\tau_{n}$ is the volume of the unit ball in $R^{n}$.
\end{abstract}

1. Introduction. It is a fundamental result of Calkin [6] (see also [12], [23]) that all nontrivial two-sided ideals of operators on a separable Hilbert space can be indexed by a particular set of vector spaces of sequences. If $Y$ is the sequence space, then an operator $A \in I_{Y}$, the associated ideal, if and only if the singular values of $A,\left\{\mu_{n}(A)\right\}_{n=1}^{\infty}$ (these are the eigenvalues of $|A|=\left(A^{*} A\right)^{1 / 2}$ arranged so that $\left.\mu_{1} \geqslant \mu_{2} \geqslant \cdots\right)$ is an element of the sequence space $Y$. Among the allowed sequence spaces are the $l^{p}$ spaces and the more general Lorentz spaces $l(p, q)[18],[15]$. The ideal $I_{p}$ associated to $l^{p}$ is precisely the ideal introduced by von Neumann and Schatten [24]. These ideals have been quite useful in a variety of analytic considerations (for example, recent applications to problems of mathematical physics, see Deift-Simon [8], Seiler [26] or SeilerSimon [27]); of especial use has been the complex interpolation theory for these ideals, developed essentially by Kunze [17] (see also [13], [21]). The more general ideals $I_{p, q}$ associated to $l(p, q)$ have found some applications to a rather special problem in operator theory [1], [2] but they do not appear to have found application to any wider class of analytic problems. Our goal in this paper is to develop the theory of the weak trace ideals $I_{p}^{w}$ associated to the weak$l_{p}$ spaces $l(p, \infty)$, especially their interpolation theory.

Let us try to explain why the spaces $I_{p}^{w}$ arise naturally. A positive selfadjoint operator $A$ lies in $I_{p}^{w}$ if and only if $A$ is compact and its eigenvalues $\lambda_{n}$ obey

Received by the editors October 17, 1975. 81 A09.

AMS (MOS) subject classifications (1970). Primary 47D99, 35P20; Secondary 41A05,

Key words and phrases. Weak trace ideals, Schrödinger operators.

(1) A. Sloan Foundation Fellow; research partially supported by N.S.F. under Grant GP-39048 A\#1. 


$$
\#\left\{n \mid \lambda_{n}(A) \geqslant \alpha\right\} \leqslant c \alpha^{-p}
$$

(the smallest $c$ allowed is called the $I_{p}^{w}$ norm, $\|A\|_{p, w}$, of $A$; it is not a norm however!). Now consider a positive operator $B$ which is unbounded with compact resolvent so that

$$
\#\left\{n \mid \lambda_{n}(B) \leqslant \alpha\right\} \sim c \alpha^{p} .
$$

Such a situation is quite common; for example a celebrated theorem of Weyl asserts that $-\Delta_{\Omega}$, the Dirichlet boundary condition Laplacian for some region $\Omega \subset \mathbf{R}^{n}$, has the property (2) with $p=n / 2$. If (2) holds and $p \geqslant 1$ then $A=$ $(B+1)^{-1}$ lies in $I_{p}^{w}$ and this is the best information one can give for $A$ in terms of $I_{p, q}$ spaces.

Our own interest in the $I_{p}^{w}$ spaces arose in a context more complex than that of the last paragraph, but one closely related to it. For any "potential" $V \in L^{n / 2}\left(\mathbf{R}^{n}\right)+L^{\infty}\left(\mathbf{R}^{n}\right)$, let $-\Delta+V$ be defined as a form sum (see Faris [10], [11], Reed-Simon [21] or Simon [28]). Let $N(V)$ be the number of "bound states" of $-\Delta+V$, i.e., the number of independent negative eigenfunctions for $-\Delta+V$. Martin [19] (see also [33]) proved a beautiful result for $V$ 's which were Hölder continuous with compact support:

$$
\lim _{\lambda \rightarrow \infty} N(\lambda V) / \lambda^{n / 2}=(2 \pi)^{-n} \tau_{n} \int\left(V_{-}(x)\right)^{n / 2} d^{n} x
$$

where $V_{-}$is the negative part of $V$ and $\tau_{n}$ is the volume of the unit sphere in $\mathbf{R}^{n}$. (3) is an especially beautiful result because of its connection with the relationship between quantum mechanics and classical mechanics; for the right side of (3) is just $(2 \pi)^{-n}$ Vol where $\mathrm{Vol}$ is the volume of phase space where the classical energy $p^{2}+V(x)$ is negative. On the other hand, since $-\Delta+\lambda V=$ $\lambda\left(-\lambda^{-1} \Delta+V\right)$ the left side of (3) represents the number of bound states of a quantum system multiplied by $h^{n}$ in the limit as $h \rightarrow 0$. Thus (3) gives meaning to the statement that in the classical limit, the number of bound states of a quantum system is given by the volume of phase space divided by $h^{n}$ (where $h=$

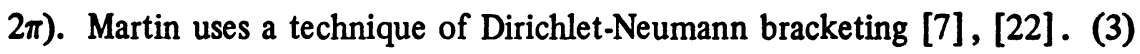
has been proven independently by Tamura [33] using Green's function techniques. Tamura eliminates a certain amount of smoothness on $V$ but still requires it to go to zero at infinity, be smooth near infinity and have only negative singularities.

One way of proving (3) for more general $V$ s is by an approximation argument. This requires a bound on $\overline{\lim }_{\lambda \rightarrow \infty} N(\lambda V) / \lambda^{n / 2} \equiv N_{\infty}(V)$ with $N_{\infty}(V)$ if $V$ is sufficiently small. We conjecture the bound

$$
N(V) \leqslant c_{n} \int\left|V_{-}(x)\right|^{n / 2} d^{n} x
$$


for $n \geqslant 3$ and will reduce this to a conjecture on certain integral operators lying in $I_{n}^{w}$. Using interpolation theory we will prove that

$$
N(V) \leqslant C_{n, \epsilon}\left[\left\|V_{-}\right\|_{n / 2+\epsilon}+\left\|V_{-}\right\|_{n / 2-\epsilon}\right]^{n / 2}
$$

for $n \geqslant 3, \epsilon>0$, where $\|f\|_{p}^{p}=\int|f|^{p} d^{n} x$. (4) would allow us to extend (3) to all $V \in L^{n / 2}$. Using (5), we will extend (3) to all $V \in L^{n / 2+\epsilon} \cap L^{n / 2-\epsilon}$.

We will make extensive use of the following result which is a special case of an interpolation theorem of Hunt [14], [15].

THEOREM 1.1. Let $p_{1}<p_{2}, q_{1}<q_{2}, 0<t<1$, and define $p_{t}$, $q_{t}$ by $p_{t}^{-1}$ $=t p_{1}^{-1}+(1-t) p_{0}^{-1} ; q_{t}^{-1}=t q_{1}^{-1}+(1-t) q_{0}^{-1}$. Let $T$ be a bounded linear map from $L^{p_{t}}(M, d \mu)$ to $L^{q_{t}}(N, d \nu)$ for $t=0,1$ with norm $N_{t}$. Then $T$ takes $L_{w}^{p_{t}}$ to $L_{w}^{q_{t}}$ for $0<t<1$ and

$$
\|T f\|_{q_{t}, w} \leqslant C \max \left(N_{0}, N_{1}\right)\|f\|_{p_{t}, w}
$$

for a constant $C$ depending only on $p_{i}, q_{i}$ and $t$.

In (6), $\|\cdot\|_{p, w}$ is defined by:

$$
\|f\|_{p, w}=\left[\sup _{t} t^{p} \mu\{x|| f(x) \mid \geqslant t\}\right]^{1 / p} .
$$

Since Hunt's more general theorem includes the Marcinkiewicz theorem, his proof is rather subtle. The special case Theorem 1 is very elementary and we provide a proof for the reader's convenience in Appendix 1.

The content of this paper is the following: In $\$ 2$, we present a few properties of $I_{p}^{w}$; in $\S 3$ we prove interpolation theorems for $I_{p}$ and $I_{p}^{w}$ by reducing them to interpolation theorems for $l_{p}$ and $l_{p}^{w}$ (we explore this idea further in Appendix 2). In \$4 we make some conjectures about integral operators and prove some results slightly weaker than these conjectures. The proof of (5) and application to prove (3) for $L^{n / 2-\epsilon} \cap L^{n / 2+\epsilon}$ appear in $\$ 5$.

It is a pleasure to thank $\mathrm{G}$. Bennett for valuable correspondence and $\mathrm{E}$. Seiler for valuable discussions.

2. Properties of weak trace ideals. Throughout, we fix a Hilbert space:

Definition. Let $A$ be a compact operator. The singular values of $A$, $\mu_{n}(A)$, are the eigenvalues of $|A|=\sqrt{A^{*} A}$ listed according to $\mu_{1} \geqslant \mu_{2} \geqslant \cdots$.

We will need the following inequalities:

$$
\begin{gathered}
\mu_{n}(B A)=\mu_{n}(A B) \leqslant\|B\| \mu_{n}(A), \quad \text { all bounded } B, \\
\mu_{n+m-1}(A+B) \leqslant \mu_{n}(A)+\mu_{m}(B), \\
\mu_{n+m-1}(A B) \leqslant \mu_{n}(A) \mu_{m}(B) .
\end{gathered}
$$


(8) is elementary; (9) and (10) are inequalities of Fan [9] following from minmax considerations; see, e.g. [13].

Definition. A compact operator $A$ is said to lie in $I_{p}$ if and only if $\Sigma_{n=1}^{\infty} \mu_{n}(A)^{p}<\infty$. $\|A\|_{p}$ is defined by:

$$
\|A\|_{p} \leqslant\left(\sum_{n=1}^{\infty} \mu_{n}(A)^{p}\right)^{1 / p} .
$$

Definition. A compact operator $A$ is said to lie in $I_{p}^{w}$ if and only if $\mu_{n}(A) \leqslant c n^{-1 / p}$ for some $c$. $\|A\|_{p}^{w}$ is defined by:

$$
\|A\|_{p, w}=\sup _{n}\left|n^{1 / p_{\mu}} \mu_{n}(A)\right| \text {. }
$$

REMARKS. (1) Since $\mu_{n}$ is monotone decreasing, $\mu_{n}(A) \leqslant c n^{-1 / p}$ if and only if $\#\left\{m \mid \mu_{m}(A) \leqslant \alpha\right\} \leqslant c^{p} \alpha^{-p}$ which is the more usual definition of $l_{p, w}$.

(2) $\|\cdot\|_{p, w}$ is not a norm but since $\|\cdot\|_{p, w}$ on $l_{p, w}$ is equivalent to a norm if $p \neq 1[31], I_{p}^{w}$ with the topology defined by $\|\cdot\|_{p, w}$ is equivalent to a symmetric normed ideal in the sense of [23], [13].

THEOREM 2.1. (a) $I_{p}$ is an ideal and moreover $\|A B\|_{p} \leqslant\|A\|\|B\|_{p}$; $\|A+B\|_{p} \leqslant\|A\|_{p}+\|B\|_{p} ;\|A B\|_{p} \leqslant\|A\|_{q}\|B\|_{r}$ where $p^{-1}=q^{-1}+r^{-1}$.

(b) $I_{p}^{w}$ is an ideal and moreover $\|A B\|_{p, w} \leqslant\|A\|\|B\|_{p, w} ;\|A+B\|_{p, w}$ $\leqslant 2^{1 / p}\left(\|A\|_{p, w}+\|B\|_{p, w}\right) ;\|A B\|_{p, w} \leqslant 2^{1 / p}\|A\|_{q, w}\|B\|_{r, w}$ where $p^{-1}=q^{-1}+$ $r^{-1}$.

REMARK. The final inequality is intended to indicate that if $A \in I_{q}^{w}, B \in$ $I_{r}^{w}$, then $A B \in I_{p}^{w}$.

Proof. (a) is standard; (b) holds if we prove the inequalities. These follow from (8)-(10). For example, by (10):

$$
\mu_{2 n}(A B) \leqslant\|A\|_{q, w}\|B\|_{r, w} n^{-1 / q}(n-1)^{-1 / r} \leqslant 2^{1 / p}\|A\|_{q, w}\|B\|_{r, w}(2 n)^{-1 / p}
$$

and similarly for $\mu_{2 n-1}(A B)$. $\square$

Let $B$ denote the family of orthonormal sequences in $H$. On a sequence $\|\cdot\|_{p}$ and $\|\cdot\|_{p, w}$ denote the usual $l_{p}$ and $l_{p}^{w}$ norm.

THEOREM 2.2. (a) $\left.\|A\|_{p}=\sup _{\{\psi}\right\}\{\varphi\} \in B\left\|\left(\psi_{n}, A \varphi_{n}\right)\right\|_{p}$.

(b) Let $p \neq 1$. Then for a suitable $C_{p}>0$ :

$$
C_{p} \sup _{\{\varphi\}\{\psi\} \in 6}\left\|\left(\psi_{n}, A \varphi_{n}\right)\right\|_{p, w} \leqslant\|A\|_{p, w} \leqslant \sup _{\{\varphi\}\{\psi\}}\left\|\left(\psi_{n}, A \varphi_{n}\right)\right\|_{p, w} .
$$

Próof. The canonical expansion for $A$ [20] asserts that

$$
A=\sum_{n=1}^{\infty} \mu_{n}(A)\left(\varphi_{n}, \cdot\right) \psi_{n}
$$


for suitable $\left\{\varphi_{n}\right\},\left\{\psi_{n}\right\} \in B$. Thus for any norm on sequences,

$$
\left\|\mu_{n}(A)\right\| \leqslant \sup _{\varphi, \psi \in B}\left\|\left(\psi_{n}, A \varphi_{n}\right)\right\|
$$

For any $f, g \in B$ :

$$
\left(f_{n} A g_{n}\right)=\sum_{m=1}^{\infty} a_{m n} \mu_{m}(A)
$$

where $a_{m n}=\left(\varphi_{m}, f_{n}\right)\left(g_{n}, \psi_{m}\right)$. A simple application of Bessel's inequality shows [29] that $a_{m n}$ is doubly substochastic

$$
\begin{aligned}
& \sum_{m=1}^{\infty}\left|a_{m n}\right| \leqslant 1, \\
& \sum_{n=1}^{\infty}\left|a_{m n}\right| \leqslant 1 .
\end{aligned}
$$

By (13), the matrix $\left\{a_{m n}\right\}$ defines a contraction on $l_{1}$ and $l_{\infty}$. Thus by the Reisz-Thorin interpolation theorem, it defines a contraction on each $l_{p}$ and by Hunt's theorem, Theorem 1 , a map of norm $d_{p}$ (independently of $\left\{a_{m n}\right\}$ ). It follows that

$$
\begin{gathered}
\left\|\left(\psi_{n}, A \varphi_{n}\right)\right\| \leqslant\|A\|, \\
\left\|\left(\psi_{n}, A \varphi_{n}\right)\right\|_{p, w} \leqslant d_{p}\|A\|_{p, w} .
\end{gathered}
$$

Theorem 2.3. Let $p \geqslant 2 . A \in I_{p}^{w}\left(\right.$ resp. $\left.I_{p}\right)$ if and only if $A^{*} A \in I_{p / 2}^{w}$ (resp. $\left.I_{p / 2}\right)$ and

$$
\|A\|_{p, w}^{2}=\left\|A^{*} A\right\|_{p, w} .
$$

Proof. $\mu_{n}\left(A^{*} A\right)=\mu_{n}(A)^{2}$ so the result is trivial. 口

3. Interpolation theorems for $I_{p}^{w}$. There is a general metatheorem which we discuss and prove in Appendix 2 which says that any interpolation theorem on symmetrically normal sequence spaces extends to the ideals indexed by these spaces. I will illustrate these ideas by proving Hunt's interpolation theorem for trace ideals:

THEOREM 3.1. Let $T$ be a linear transformation from the finite rank operators on a Hilbert space $H_{1}$ to the bounded operators on a Hilbert space $H_{2}$. Suppose that $p_{1}<p_{2}, q_{1}<q_{2}$ and that $T$ maps $I_{p_{1}}$ to $I_{q_{1}}$ and $I_{p_{2}}$ to $I_{q_{2}}$ with $\|T(A)\|_{q_{i}} \leqslant C\|A\|_{p_{i}}$. Then, for any $t \in(0,1)$, T maps $I_{p_{t}}^{w}$ to $I_{q_{t}}^{w}, p_{t}^{-1}=$ $t p_{1}^{-1}+(1-t) p_{0}^{-1}$ and $q_{t}^{-1}=t q_{1}^{-1} t(1-t) q_{0}^{-1}$. Moreover

$$
\|T(A)\|_{q_{t}, w} \leqslant C D\|A\|_{p_{t}, w}
$$


where $D$ only depends on $p_{i}, q_{i}$ and $t$ (and is the constant in the usual Hunt theorem).

In proving Theorem 3.1, we will also prove:

THEOREM 3.2. Theorem 3.1 remains true if $H_{1}$ is replaced by a finite measure space $(M, d \mu)$ and $I_{p}\left(\right.$ resp. $\left.I_{p}^{w}\right)$ by the corresponding $L^{p}$ (resp. $\left.L^{p, w}\right)$ spaces.

Proof. Fix two orthonormal sets $\{\varphi\},\{\psi\}$ in $H_{2}$. Let $T_{\varphi, \psi}: L^{p}(M, d \mu)$ $\rightarrow l_{\infty}$ be defined by

$$
T_{\varphi, \psi}(f)_{n}=\left(\varphi_{n}, T(f) \psi_{n}\right) .
$$

Then by hypothesis and the bound

$$
\left\|\left(\varphi_{n}, A \psi_{n}\right)\right\|_{p} \leqslant\|A\|_{p}
$$

$T_{\varphi, \psi}$ is bounded from $L^{p_{i}}$ to $l_{q_{i}}(i=1,2)$ with bound $C$. Thus by the usual Hunt theorem $T_{\varphi, \psi}$ is bounded from $L_{w}^{p_{t}}$ to $l_{q_{t}, w}$ with norm $C D$ where $D$ is a constant independent of $\varphi, \psi$ and only dependent on $p_{i}, q_{i}$ and $t$. Thus, by Theorem 2.2(b):

$$
\|T(f)\|_{q_{t}, w} \leqslant \sup _{\varphi, \psi}\left\|T_{\varphi, \psi}(f)\right\|_{q_{t}, w} \leqslant C D_{1}\|f\|_{p_{t}, w} \quad \square
$$

Proof of Theorem 3.1. Fix $A \in I_{p_{t}}^{w}$. We will prove that

$$
\|T(A)\|_{q_{t}, w} \leqslant C D\|A\|_{p_{t}, w} .
$$

For let $A=\Sigma \mu_{n}\left(\varphi_{n}, \cdot\right) \psi_{n}$ be the canonical expansion for $A$. For any finite sequence, define $S(\{\lambda\})$ by

$$
S(\{\lambda\})=T\left(\sum_{n} \lambda_{n}\left(\varphi_{n}, \cdot\right) \psi_{n}\right)
$$

By hypothesis, $S$ takes $l_{p_{i}}$ into $I_{q_{i}}$ with norm bounded by $C$, so by Theorem 3.2 , it takes $l_{p_{t}, w}$ into $I_{q_{t}, w}$ with

Thus

$$
\left\|S\left(\left\{\lambda_{n}\right\}\right)\right\|_{q_{t}, w} \leqslant C D\|\{\lambda\}\|_{p_{t}, w} .
$$

$$
\|T(A)\|_{q_{t}, w} \equiv\left\|S\left(\left\{\mu_{n}\right\}\right)\right\|_{q_{t}, w} \leqslant C D\left\|\left\{\mu_{n}\right\}\right\|_{p_{t}, w}=C D\|A\|_{p_{t}, w} . \quad \square
$$

4. Some conjectures.

ConJeCtuRe 1. Let $2<p<\infty$. For functions $f, g$ on $\mathbf{R}^{n}$ define an operator $A_{f, g}$ on $L^{2}\left(R^{n}, d^{n} x\right)$ by the integral kernel $f(x-y) g(y)$. Then, if $g \in$ $L^{p}\left(R^{n}, d^{n} x\right)$ and $f \in L_{w}^{p^{\prime}}\left(\mathbf{R}^{n}, d^{n} x\right)$ (where $\left.p^{\prime}=\left(1-p^{-1}\right)^{-1}\right), A_{f, g} \in I_{p}^{w}$ and

$$
\left\|A_{f, g}\right\|_{p, w} \leqslant C\|f\|_{p^{\prime}, w}\|g\|_{p} .
$$


This is the main conjecture of this paper. Its truth, as we shall see, would lead to the Schrödinger operator bound (4). As support for the conjecture we note several results very close to it. The first two are certainly not new. The fourth is new and will lead to the bound (5).

Proposition 4.1. If $f \in L_{w}^{p^{\prime}}$ and $g \in L_{w}^{p}(2<p<\infty)$, then $A_{f, g}$ is a bounded operator; $\left\|A_{f, g}\right\| \leqslant C\|f\|_{p^{\prime}, w}\|g\|_{p, w}$.

Proof. This follows from the generalized Sobolev inequality [32], $p>q$, $p<\infty, q>1$ :

$$
\|h[f *(g k)]\|_{1} \leqslant\|h\|_{q^{\prime}}\|f\|_{p^{\prime}, w}\|g\|_{p^{\prime}, w}\|k\|_{q}
$$

(15) is proven first without the $w$ by appealing to Young's and Hölder's inequalities and then by using the Marcinkiewicz and Hunt interpolation theorems. $\square$

REMARKS. (1) For the case $q=2$ of interest, (15) implies the operator inequality on $L^{2}\left(\mathbf{R}^{n}, d^{n} x\right)$ :

$$
|x|^{-2 \alpha} \leqslant C_{\alpha, n}(-\Delta)^{\alpha}
$$

so long as $\alpha<1 / 2 n$. Conversely, using the symmetric rearrangement theorem [4], (16) implies (15) with $q=2$.

(2) We will see below that when $g \in L^{p}, A_{f, g}$ is compact.

Proposition 4.2 (Seiler-Simon [27], T. Kato (UNPUblished)). If $2 \leqslant p \leqslant \infty$ and if $f \in L^{p^{\prime}}, g \in L^{p}$, then $A_{f, g}$ is in $I_{p}$ and $\left\|A_{f, g}\right\|_{p} \leqslant C\|f\|_{p}\|g\|_{p}$.

Proof. This is easy for $p=2$ or $p=\infty$ and follows for general $p$ by complex interpolation.

Corollary 4.3. If $2<p<\infty, f \in L_{w}^{p^{\prime}}$ and $g \in L^{p}$, then $A_{f, g}$ is compact.

Proof. Since $\left\|A_{f, g}\right\| \leqslant C\|f\|_{p^{\prime}, w}\|g\|_{p}$, it suffices to prove it if $g \in L^{1} \cap$ $L^{\infty}$. But, in that case $f=f_{1}+f_{2}$ with $f_{1} \in L_{w}^{p^{\prime}-\epsilon}, f_{2} \in L^{p^{\prime}+\epsilon}$ and $A_{f_{i}, g}$ is in an $I_{p}$ space and so compact.

Proposition 4.4. Let $2<p<\infty, f \in L_{w}^{p^{\prime}}, g \in L^{p+\epsilon} \cap L^{p-\epsilon}$. Then $A_{f, g}$ $\in I_{p^{\prime}}^{w}$ and $\left\|A_{f, g}\right\|_{p^{\prime}, w} \leqslant C_{\epsilon, p}\|f\|_{p^{\prime}, w}\left(\|g\|_{p+\epsilon}+\|g\|_{p-\epsilon}\right)$.

Proof. Let $\alpha=(p+\epsilon)^{\prime}, \beta=(p-\epsilon)^{\prime}$. Then since $g \in L^{p+\epsilon}, f \rightarrow A_{f, g}$ maps $L^{\infty}$ into $I_{\alpha}$ by Proposition 4.2. Similarly it moves $L^{p}$ into $I_{p}$. The norms are bounded by $C\|g\|_{p+\epsilon}$ and $C\|g\|_{p-\epsilon}$ respectively. By Theorem 3.2, it maps $L_{w}^{p^{\prime}}$ into $I_{p^{\prime}, w}$. The norm relation is an easy consequence of the norm relations and the linearity of $A_{f, g}$ in $g$. $\quad$ 
In attempting to prove Conjecture 1, several related questions have arisen:

Conjecture 2. Let $f$ and $g$ be positive functions on $\mathbf{R}^{n}$. Let $f^{*}, g^{*}$ denote the spherical rearrangement of $f, g$. For any $p \geqslant 2$, we have $\left\|A_{f, g}\right\|_{p} \leqslant\left\|A_{f^{*}, g}\right\|_{p}$ and $\left\|A_{f, g}\right\|_{p, w} \leqslant\left\|A_{f *, g} *\right\|_{p, w}$.

CONJECTURE 3. Let $f$ and $g$ be complex valued functions. Then for any $p \geqslant$ 2, we have $\left\|A_{f, g}\right\|_{p} \leqslant\left\|A_{|f|,|g|}\right\|_{p}$ and $\left\|A_{f, g}\right\|_{p, w} \leqslant\left\|A_{|f|,|g|}\right\|_{p, w}$.

Proposition 4.5. Conjectures 2 and 3 are true in the $I_{p}$ (rather than $I_{p}^{w}$ ) case when $p$ is any even integer.

PROOF. In that case, $\left\|A_{f, g}\right\|_{p}^{p}=\operatorname{Tr}\left(\left(A^{*} A\right)^{1 / 2}\right)$ is given by an explicit integral. Conjecture 3 is trivial and Conjecture 2 is a consequence of the spherical rearrangement theorem of Brascamp, Lieb and Luttinger [4].

We suspect Conjecture 2 is false in case $p<2$. If $\mathbf{R}^{n}$ is replaced by a torus so that one can take $g=1$, then $A_{f}$ is trace class if and only if $\Sigma|\hat{f}(n)|<\infty$. It is easy to find $f$ with $A_{f}$ not $I_{p}$ for $p<2$ but $A_{|f|}$ and $A_{f^{*}}$ trace class.

5. Application to Schrödinger operators. The key to applying $I_{p}$ methods to the study of $N(V)$ is the following result of Birman [3] and Schwinger [25]:

THEOREM 5.1 ([3], [25]). Let $n \geqslant 3$. Let $V \leqslant 0$. Then $N(V)$ is equal to the number of eigenvalues of the integral operator $\Omega(V)$ with kernel $c_{n}|V(x)|^{1 / 2}|x-y|^{-n+2}|V(y)|^{1 / 2}$, which are larger than +1 .

REMARK. $c_{n}$ is chosen so that $\Omega(V)$ is just $|V|^{1 / 2}(-\Delta)^{-1}|V|^{1 / 2}$. The key idea in the proof (see also [28]) is that $E<0$ is an eigenvalue of $-\Delta+\lambda V$ if and only if $\lambda^{-1}$ is an eigenvalue of $|V|^{1 / 2}(-\Delta-E)^{-1}|V|^{1 / 2} . n \geqslant 3$ is critical for $(-\Delta)^{-1}$ to define an integral operator.

Now let $\omega(V)$ be the integral operator with kernel $d_{n}(x-y)^{-n+1}|V(y)|^{3 / 2}$ with $d_{n}$ chosen so that $\omega(V)=(-\Delta)^{-1 / 2}|V|^{1 / 2}$.

Proposition 5.2. (i) Let $V \leqslant 0 . N(\lambda V) \leqslant c \lambda^{n / 2}$ for all $\lambda$ if and only if $\omega(V) \in I_{n}^{w}$ with $\|\omega(V)\|_{n, w}^{n} \leqslant c$.

(ii) Let $X$ be a Banach space of potentials in which $C_{0}^{\infty}$ is dense with $\|V\|_{X}$ $\geqslant d\|V\|_{n / 2}$. Suppose that, for any $V \in X, N(V) \leqslant c\|V\|_{X}^{n / 2}$. Then for any $V \in X$ :

$$
\lim _{\lambda \rightarrow \infty} N(\lambda V) / \lambda^{n / 2}=(2 \pi)^{-n} \tau_{n} \int\left[V_{-}(x)\right]^{n / 2} d^{n} x .
$$

Proof. (i) By Theorem 5.1,N( $\lambda V) \leqslant c \lambda^{n / 2}$ if and only if the number of eigenvalues of $\Omega(V)$ larger than $\lambda^{-1}$ is bounded by $c \lambda^{n / 2}$. This is true if and only if $\Omega(V) \in I_{n / 2}^{w}$ and $\|\Omega(V)\|_{n / 2, w}^{n / 2} \leqslant c$. Since $\Omega(V)=\omega(V)^{*} \omega(V)$, the proof is completed by appealing to Theorem 2.3.

(ii) Let $A$ be an arbitrary selfadjoint operator which is bounded from below. 
Let $n(A)$ be the dimension of the spectral projection $P_{(-\infty, 0)}$. Then

$$
n(A+B) \leqslant n(A)+n(B)
$$

if $Q(A) \cap Q(B)$ is dense. $A+B$ is defined as a form sum $(Q(\cdot)=$ quadratic form domain of $)$. We prove (17) in Lemma 5.3.

Now, given $V, W \in X$, by (17), we have:

$$
\begin{aligned}
N(V) & =n(-\Delta+V) \\
& \leqslant n(-\epsilon \Delta+(V-W))+n(-(1-\epsilon) \Delta+W) \\
& =N\left(\epsilon^{-1}(V-W)\right)+N\left((1-\epsilon)^{-1} W\right) .
\end{aligned}
$$

By hypothesis, given $V \in X$ and $\epsilon$, we can find $W \in C_{0}^{\infty}$ with $\|V-W\|_{x} \leqslant \epsilon^{2+n / 2}$ and $\int\left|V_{-}^{n / 2}-W_{-}^{n / 2}\right| \leqslant O\left(\epsilon^{1+n / 2}\right)$. Then:

$$
\varlimsup \frac{N(\lambda V)}{\lambda^{n / 2}} \leqslant O(\epsilon)+\lim \frac{N(\lambda W)}{\lambda^{n / 2}}(1-\epsilon)^{-n / 2}
$$

by (18);

$$
\leqslant O(\epsilon)+(2 \pi)^{-n} \tau_{n} \int V_{-}^{n / 2}
$$

by (3) (Martin [19], Tamura [33]). Interchanging $V$ and $W$ in (18) we see that

$$
\underline{\lim } \frac{N(\lambda V)}{\lambda^{n / 2}} \geqslant O(\epsilon)+(2 \pi)^{-n} \tau_{n} \int V_{-}^{n / 2} .
$$

Since $\epsilon$ is arbitrary, we are done.

Lemma 5.3. Let $A$ and $B$ be selfadjoint operators with $Q(A) \cap Q(B)$ dense. Define $A+B$ as a form sum. Then

$$
n(A+B) \leqslant n(A)+n(B) .
$$

Proof. Without loss, we can suppose that $n(A)<\infty, n(B)<\infty$. If $n(A+B)>n(A)+n(B)$, then we could find a $\varphi$ with $(\varphi,(A+B) \varphi)<0$ so that $\varphi$ is orthogonal to the $n(A)$-eigenvectors of $A$ and $n(B)$-eigenvectors of $B$ associated to negative eigenvalues. But then $(\varphi, A \varphi) \geqslant 0 ;(\varphi, B \varphi) \geqslant 0$. This contradiction proves that $n(A+B) \leqslant n(A)+n(B)$.

As immediate corollaries of Proposition 5.2 we have:

THEOREM 5.4. If Conjecture 1 holds, then (4) is true and (3) holds for all $V \in L^{n / 2}$.

By Propositions 5.2 and 4.4:

THEOREM 5.5. The bound (5) holds and (3) extends to all $V \in L^{n / 2+\epsilon} \cap$ $L^{n / 2-\epsilon}$ for any $\epsilon>0$. 
REMARK. Conversely if the bound (4) holds, then for any $V$ with $V \leqslant 0$, $\|\omega(V)\|_{n / 2, w} \leqslant c\|V\|_{n / 2}$ and so, for any $V,\|\omega(V)\|_{n / 2, w} \leqslant 2 c\|V\|_{n / 2}$. Thus the truth of (4) and Conjectures 2 and 3 would imply Conjecture 1 for $p=n / 2$. We feel that Conjecture 1 is "substantially equivalent" to equation (4). This bespeaks the "naturalness" of the methods we describe.

Appendix 1. Hunt's interpolation theorem. In this appendix we describe a proof of Theorem 1.1. We do this not only because we wish this paper to be self-contained, but also because the proof of this special use of Hunt's more general theorem is much simpler than the general case. The proof we give is not readily available; we learned it several years ago from E. Nelson.

Lemma A.1.1. Let $p_{0}<p_{1}, 0<t<1$, and $p_{t}^{-1}=t p_{1}^{-1}+(1-t) p_{0}^{-1}$. Then there exists a constant $C$ (depending only on $t, p_{1}$ and $p_{2}$ ) so that, for any $f \in L_{w}^{p_{t}}$, and any $\lambda \in(0, \infty)$, there are $f_{0}^{(\lambda)}$ and $f_{1}^{(\lambda)}$ so that $f=f_{0}^{\lambda}+f_{1}^{(\lambda)} ; f_{i}^{(\lambda)}$ $\in L^{p_{i}}$ and

$$
\left\|f_{i}^{(\lambda)}\right\|_{p_{i}}^{p_{i}} \leqslant c\|f\|_{p_{t}, w}^{p_{t} \lambda^{\left(p_{i}-p_{t}\right)}}
$$

Proof. Let $f_{\lambda}^{(0)}(x)$ be 0 or $f(x)$ depending on whether $|f(x)|$ is $\leqslant \lambda$ or $>\lambda$. Let $f_{\lambda}^{(1)}(x)$ be 0 or $f(x)$ depending on whether $|f(x)|$ is $>\lambda$ or $\leqslant \lambda$. a

Lemma A.1.2. Fix $p_{0}, p_{1}, t, p_{t}$ as in Lemma A.1.1. Then there exists $D$ (depending only on $t, p_{1}$ and $p_{2}$ ) so that for any function $f$ with the property that for any $\lambda \in(0, \lambda), f=f_{0}^{(\lambda)}+f_{1}^{(\lambda)}$ with

$$
\left\|f_{i}^{(\lambda)}\right\|_{p_{i}}^{p_{i}} \leqslant \alpha \lambda^{p_{i} p_{t}}
$$

$f$ is in $L_{w}^{p_{t}}$ and $\|f\|_{p_{t}, w}^{p_{t}} \leqslant D \alpha$. tion,

Proof. Let $m_{f}(\mu)=$ meas $\{x|| f(x) \mid>\mu\}$. Then by an elementary calcula-

$$
m_{g}(\mu) \leqslant \mu^{-p}\|g\|_{p}^{p}
$$

Thus

$$
m_{f_{i}(\lambda)}\left(\frac{\lambda}{2}\right) \leqslant 2^{p_{i} p_{t}} \alpha \lambda^{p_{t}}
$$

Since $m_{f+g}(\lambda) \leqslant m_{f}(\lambda / 2)+m_{g}(\lambda / 2)$, the result follows. $\square$

ProOF OF THEOREM 1.1. We rewrite condition (19) by taking $p_{i}$ th roots and letting $\mu^{\beta}=\lambda$ with $\beta=p_{t}^{-1}\left[p_{0}^{-1}-p_{1}^{-1}\right]^{-1}$. Thus we see that (19) is replaced by requiring a breakup $f=g_{\mu}+h_{\mu}$ with

$$
\left\|g_{\mu}\right\|_{p_{0}} \leqslant \alpha \mu^{-t}, \quad\left\|h_{\mu}\right\|_{p_{1}} \leqslant \alpha \mu^{1-t}
$$


We can make a similar change in Lemma A.1.1. Suppose $f \in L_{w}^{p_{t}}$ with $\|f\|_{p_{t}, w}$ $\leqslant 1$. Then we can write $f=g_{\mu}+h_{\mu}$ with

$$
\left\|g_{\mu}\right\|_{p_{0}} \leqslant c \mu^{-t} ; \quad\left\|h_{\mu}\right\|_{p_{1}} \leqslant \mu^{1-t}
$$

Thus $T f=T g_{\mu}+T h_{\mu}$ and

$$
\left\|T g_{\mu}\right\|_{q_{0}} \leqslant C N_{0} \mu^{-t} ; \quad\left\|T h_{\mu}\right\|_{q_{1}} \leqslant C N_{1} \mu^{1-t}
$$

so $T f \in L_{w}^{q_{t}}$ and $\|T f\|_{q_{t}, w} \leqslant C D \max \left(N_{0}, N_{1}\right)$. (6) follows by homogeneity.

Appendix 2. Interpolation theorems for symmetrically normed ideals of operators. Among the operator ideals are the symmetrically normed ideals studied in [13], [23]. These correspond to sequence spaces which are Banach spaces whose norm $\Phi\left(a_{1}, a_{2}, \ldots\right)$ has the following properties:

(i) $\Phi\left(a_{i}\right)=\lim _{n \rightarrow \infty} \Phi\left(a_{1}, \ldots, a_{n}, 0,0, \ldots, 0, \ldots\right)$.

(ii) $\Phi\left(a_{i}\right)=\Phi\left(\left|a_{i}\right|\right)$.

(iii) $\left\langle a_{1}, \ldots, a_{n}\right\rangle \rightarrow \Phi\left(a_{1}, \ldots, a_{n}, 0,0, \ldots\right)$ is a symmetric function on $\mathrm{C}^{n}$. We denote the corresponding ideal by $I_{\Phi}$, i.e., $A \in I_{\Phi}$ if and only if its singular values $\mu_{n}(A)$ obey $\Phi\left(\mu_{n}(A)\right)<\infty$. Among the $I_{\Phi}$ are the $I_{p}$-ideals, the weak trace ideals $I_{p, w}$ with $p \neq 1$ with an "honest" norm equivalent to $\|\cdot\|_{p, w}$ (which is not a norm) and also Orlicz ideals corresponding to Orlicz sequence spaces [16].

In this appendix, we prove a general metatheorem which allows one to transfer interpolation theorems from symmetrically normed sequence spaces to their associated ideals. Included in this general theorem is a Reisz-Thorin theorem for $I_{p}$ [17], interpolation theorems for Orlicz ideals which follow those for Orlicz spaces [5], the theorems we prove for $I_{p, w}$ in $\S 3$, and a Marcinkiewicz theorem. The same method allows the transfer of Stein interpolation theorems [30] and "wandering analytic function theorems" [13].

The key is the following:

LEMMA A.2.1. Let $\Phi$ be a symmetric norming function. Let $B_{i j}$ be a coubly substochastic matrix, i.e., $\Sigma_{i}\left|B_{i j}\right| \leqslant 1$, all $j ; \Sigma_{j}\left|B_{i j}\right| \leqslant 1$, all $i$. Let $a \in l_{\Phi}$, the associated sequence space and define

$$
(B a)_{i}=\sum B_{i j} a_{j}
$$

Then $B a \in l_{\Phi}$ and $\Phi(B a) \leqslant \Phi(a)$.

Proof. By a simple limiting argument, we can suppose that $B$ is a finite matrix and deal with sequences $\left(a_{1}, \ldots, a_{n}, 0, \ldots\right)$. We claim that in that case we can find $B^{*}$ with $\left|B_{i j}\right| \leqslant B_{i j}^{*}$ with $B^{*}$ doubly stochastic, i.e., $\Sigma_{i} B_{i j}^{*}=$ $\Sigma_{j} B_{i j}^{*}=1$. Temporarily deferring the proof of this claim, we note that $\Phi(B a)$ $\leqslant \Phi\left(B^{*}|a|\right)$ (since $\Phi(a) \leqslant \Phi(b)$ if $\left|a_{i}\right| \leqslant\left|b_{i}\right|$; see [13]). Since $B^{*}$ is doubly 
stochastic, it is a convex combination of permutation matrices (i.e., doubly stochastic matrices with all elements 0 or 1$)$. Since $\Phi$ is convex and symmetric, we conclude that $\Phi\left(B^{*}|a|\right) \leqslant \Phi(|a|)=\Phi(a)$.

This leaves us to verify the claim about the existence about $B^{*}$. Without loss suppose that $B_{i j} \geqslant 0$. Define $B_{i j}^{*}$ inductively, defining first $B_{11}^{*}$, then $B_{12}^{*}$, $\ldots, B_{1 n}^{*}, B_{21}^{*}, \ldots, B_{n n}^{*}$ : at each stage define $B_{i j}^{*}$ to be as large as possible without destroying the double substochasticity. Thus at each stage, we increase the $i, j$ element until either the $i$ th row or the $j$ th column sums to 1 . We claim that $B^{*}$ is doubly stochastic. For, if some row, say the $i$ th, does not sum to 1 , then by the above, each column must sum to 1 . But a doubly substochastic finite matrix in which each column sums to 1 is doubly stochastic.

As in the proof of Theorem 2.2, Lemma A.2.1 immediately implies:

THEOREM A.2.2. Let $A \in I_{\Phi}$. Let $B$ denote the family of all orthonormal sequences. Then:

$$
\|A\|_{\Phi}=\sup _{\varphi, \psi \in B}\|(\varphi, A \psi)\|_{\Phi} .
$$

Given Theorem A.2.2, we can mimic the methods of $\$ 3$ to prove:

THEOREM A.2.3. Any interpolation theorem between Banach spaces remains true if all symmetrically normed sequence spaces are replaced by their corresponding operator ideals.

REMARK. By interpolation theorem, we think of 6 spaces $X_{0}, X_{1}, \tilde{X} Y_{0}$, $Y_{1}$ and $\tilde{Y}$ and $X_{0} \cap X_{1}$ (resp. $Y_{0} \cap Y_{1}$ ) dense in $X_{0}, X_{1}$ and $\widetilde{X}$ (resp. $Y_{0}, Y_{1}$, $\left.\tilde{Y}^{\prime}\right)$ so that any map from $X_{0} \cap X_{1}$ into $Y_{0}+Y_{1}$ which takes $X_{i}$ to $Y_{i}$ takes $\tilde{X}$ to $\tilde{Y}$ with a corresponding inequality on norms.

Added notes. (1). Some work related to this paper, and, in particular, a version of Proposition 5.2, appear in papers of M. S. Birman and coworkers; see Birman-Borov, Topics in Math. Phys. 5 (1972), 19-30, Birman-Solomjak, Functional Anal. Appl. 4 (1960), 265; Trans. Moscow Math. Soc. 27 (1972), 1-52; 28 (1973), 1-32.

(2) The bound (4) conjectured in this paper has been proven independently by E. Lieb (Princeton Univ. (preprint)) M. Cwickel (Institute for Advanced Study (preprint)) and M. Rosenbljum (Leningrad (in press)). Cwickel, in particular, proves our Conjecture 1.

\section{REFERENCES}

1. G. Bennett, Some ideals of operators on Hilbert space, Studia Math. (to appear).

2. G. Bennett, V. Goodman and C. M. Newman, Norms of random matrices, Indiana University (preprint).

3. M. S. Birman, On the number of eigenvalues in a quantum scattering problem, Vestnik Leningrad Univ. 16 (1961), no. 13, 163-166. (Russian) MR 25 \#2803. 
4. H. J. Brascamp, E. H. Lieb and J. M. Luttinger, A general rearrangement inequality for multiple integrals, J. Funtional Analysis 17 (1974), 227-237. MR 49 \#10835.

5. A. P. Calderón, Intermediate spaces and interpolation, Studia Math. (Ser. Spec.) Zeszyt 1 (1960), 31-34. MR 26 \#5409.

6. J. W. Calkin, Two-sided ideals and congruences in the ring of bounded operators in Hilbert spaces, Ann. of Math. (2) 42 (1941), 839-873. MR 3, 208.

7. R. Courant and D. Hilbert, Methods of mathematical physics. Vol. 1, Interscience, New York, 1953. MR 16, 426.

8. P. Deift and B. Simon, On the decoupling of finite singularities from the question of asymptotic complexness in two body quantum system, J. Functional Analysis (to appear).

9. K. Fan, Maximum properties and inequalities for the eigenvalues of completely continuous operators, Proc. Nat. Acad. Sci. U.S.A. 37 (1951), 760-766. MR 13, 661.

10. W. Faris, The product formula for semigroups defined by Friedrichs extensions, Pacific J. Math. 22 (1967), 47-70. MR 35\#5975.

11. Self-adjoint operators, Springer Lecture Notes, \#433, 1975.

12. D. J. H. Garling, On ideals of operators in Hilbert space, Proc. London Math. Soc. (3) 17 (1967), 115-138. MR 34 \#8208.

13. I. C. Gohberg and M. G. Krein, Introduction to the theory of linear non-selfadjoint operators in Hilbert space, Izdat. "Nauka", Moscow, 1965; English transl., Transl. Math. Monographs, vol. 18, Amer. Math. Soc., Providence, R. I., 1969. MR 36 \#3137; 39 \#7447.

14. R. A. Hunt, An extension of the Marcinkiewicz interpolation theorem to Lorentz spaces, Bull. Amer. Math. Soc. 70 (1964), 803-807; Addendum, ibid. 71 (1965), 396. MR 29 \#6292; 30 \#2331.

15. — On $L(p, q)$ spaces, Enseignement Math. (2) 12 (1966), 249-276. MR 36 \#6921.

16. M. A. Krasnosel'skiǐ and Ja. B. Rutickii, Convex functions and Orlicz spaces, GITTL, Moscow, 1958; English transl., Nordhoff, Groningen, 1961. MR 21 \#5144; 23\#A4016.

17. R. A. Kunze, $L_{p}$ Fourier transforms on locally compact unimodular groups, Trans. Amer. Math. Soc. 89 (1958), 519-540. MR 20 \#6668.

18. G. G. Lorentz, Some new functional spaces, Ann. of Math. (2) 51 (1950), 37-55; On the theory of spaces $\Lambda$, Pacific J. Math. 1 (1951), 411-429. MR 11, 442; 13, 470. 140-148.

19. A. Martin, Bound states in the strong coupling limit, Helv. Phys. Acta 45 (1972),

20. M. Reed and B. Simon, Methods of modern mathematical physics. Vol. I: Functional analysis, Academic Press, New York, 1972.

21. - Methods of modern mathematical physics. Vol. II: Fourier analysis, self-adjointness, Academic Press, New York, 1975.

22. - Methods of modern mathematical physics. Vol. III: Analysis of operators, Academic Press, New York, (in preparation).

23. R. Schatten, Norm ideals of completely continuous operators, Springer-Verlag, Berlin, 1960. MR 22 \#9878.

24. R. Schatten and J. von Neumann, The cross-space of linear transformations. II, III, Ann. of Math. (2) 47 (1946), 608-630; ibid. (2) 49 (1948), 557-582. MR 8, 31; $10,256$.

25. J. Schwinger, On the bound states of a given potential, Proc. Nat. Acad. Sci. U.S.A. 47 (1961), 122-129. MR 23 \#B2833.

26. E. Seiler, Schwinger functions for the Yukawa model in two dimensions with space time cut-off, Comm. Math. Phys. 42 (1975), 163-182.

27. E. Seiler and B. Simon, Bounds in the Yukawa 2 quantum field theory: upper bound on the pressure, Hamiltonian bound and linear lower bound, Comm. Math. Phys. 45 (1975), 99-114.

28. B. Simon, Quantum mechanics for Hamiltonians defined as quadratic forms, Princeton Univ. Press, Princeton, N. J., 1971. 
29. B. Simon, Notes on the infinite determinants of Hilbert space operators, Advances in Math. (to appear).

30. E. M. Stein, Interpolation of linear operators, Trans. Amer. Math. Soc. 83 (1956), 482-492. MR 18, 575.

31. Singular integrals and differentiability properties of functions, Princeton Univ. Press, Princeton, N. J., 1970. MR 44 \#7280.

32. R. S. Strichartz, Multipliers on fractional Sobolev spaces, J. Math. Mech. 16 (1967), 1031-1060. MR 35 \#5927.

33. H. Tamura, The asymptotic eigenvalue distribution for non-smooth elliptic operators, Proc. Japan Acad. 50 (1974), 19-22.

DEPARTMENTS OF MATHEMATICS AND PHYSICS, PRINCETON UNIVERSITY, PRINCETON, NEW JERSEY 08540

Current address: Department of Physics, Yeshiva University, New York, New York 10033 